\title{
Synthetic Biology Design and Analysis: a Case Study of Frequency Entrained Biological Clock
}

\begin{abstract}
Peng Yu
Department of Electrical and Computer Engineering

Department of Chemistry and Biochemistry

Xi Chen

Univerisity of Texas at Austin Univerisity of Texas at Austin

Abstract-Biological clock, a self sustaining oscillation widely discovered in many species, is important to determine many biological activities, for example, the sleep-wake cycle. Although the frequency entrainment of biological clock has long been observed, the exact biochemical mechanism for this property has not been proposed. Recent advances in synthetic biology have led researchers to engineer new or better biological systems that may or may not exist in nature. In this work, by adapting the ideas of an electronic circuit, Phase Lock Loop, we design and analyze a genetic circuit that allows the frequency entrainment of its internal biological clock, which demonstrates that knowledge and experiences accumulated in other engineering fields are great sources for the further development of synthetic biology.
\end{abstract}

\author{
David Z. Pan \\ Department of Electrical and \\ Computer Engineering
}

Department of Chemistry and Biochemistry Institute for Cellular and Molecular Biology Univerisity of Texas at Austin andy.ellington@mail.utexas.edu

\section{INTRODUCTION}

Biologic clock is a widely spread phenomenon discovered in numerous species from bacteria to human. The best studied biological clock is the circadian rhythm which is the daily cycle of physiological activities. Unlike the changes of environment caused by other factors, periodic changes of temperature, light intensity and humidity due to the rotation of the earth are highly predictable. Thus it is beneficial for organisms to anticipate and prepare for such changes by regulating the internal gene expression accordingly. For example, cyanobacteria use their circadian rhythms to regulate two biochemically incompatible processes: the photosynthesis which requires daylight, and the nitrogen fixation which does not. In most systems studied, circadian rhythms are mainly controlled by induction-repression oscillators [1], where type-A molecules are produced in the presence of type-B molecules and, once present, trigger the decay of type-B molecules. The frequency of the oscillation is determined by the kinetic parameters of the system.

One important feature of circadian rhythms is the environmental entrainment which is the ability to set the frequency (frequency entrainment) and the phase (phase entrainment) of internal oscillator according to the oscillation of external signal. Theoretically, phase entrainment (synchronization of internal and external phases) can be easily done by transiently resetting the relative concentration levels of molecules of each type upon the change of the environment. In fact, such mechanism has been observed in biology [2]. However, how to dictate the frequency of internal oscillator by the frequency of input signals remains theoretically challenging. Although many genes involved in such process have been identified by mutant screening, the exact mechanism has not been found.

The essence of frequency entrainment is to adjust the kinetic parameters of the internal oscillator by the frequency of external signal. Although the intensity of external signals (e.g. temperature, light, voltage, concentration of chemical compounds) can be sensed by causing conformational changes of proteins and affecting their kinetic properties, there is no known biochemical mechanism by which a single protein can convert the frequency of an input signal to a kinetic parameter. Thus, we will seek the solution of this problem by designing a genetic circuit. From an engineering perspective, implementation of entrainable biological clocks would offer many advantages when inputs need to change periodically. Moreover, as many synthetic oscillators [3], [4] suffer from damped amplitudes and/or unstable central values, an entrained system may improve the performance of such oscillators.

Inspired by the concepts used in an electronic circuit, Phase Lock Loop (PLL) [5], we design a Frequency Entrained Biological Clock (FEBC) which can potentially be implemented in biological systems.

\section{Overall Picture of Frequency Entrained Biological CLOCK (FEBC)}

We design the FEBC by adapting the idea of a widely used electronic circuit, Phase Lock Loop (PLL) [5]. In this section, we describe the high level design of the FEBC. The lower level details will be discussed later.

Figure 1 shows the block diagram of the FEBC, which has three major components. The component Biological Clock (BC) generates

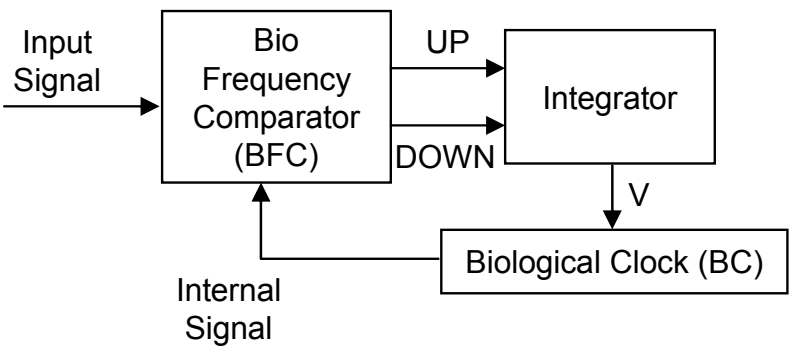

Figure 1. The Frequency Entrained Biological Clock.

an internal oscillatory signal. To adjust the $\mathrm{BC}$ frequency by the frequency of the input signal, the control parameter (V) of the BC shall be set to an appropriate value generated by the Biological Frequency Comparator (BFC) and the Integrator. The BFC compares the frequency difference between the input signal and the internal signal. It generates an UP (DOWN) signal when the input signal frequency is greater (less) than the internal signal. The Integrator generates the control value $\mathrm{V}$ corresponding to the integration of the difference between the UP and DOWN signals,

$$
\mathrm{V} \propto \int_{-\infty}^{t}(\mathrm{UP}-\mathrm{DOWN}) \mathrm{d} t .
$$

When the UP signal is present, the signal $\mathrm{V}$ increases, which leads to the increment of the frequency of the BC. When the DOWN signal is present, the signal $\mathrm{V}$ decreases, which leads to the decrement of the frequency of the $\mathrm{BC}$.

Before we present the details of each component, we will discuss the modeling of the basic biochemical reactions for the design. 


\section{Modeling Basic Biological Parts}

Among numerous mechanisms of gene regulation, we primarily use transcriptional regulation, the best studied and most successfully engineered one, in our design. The transcriptional regulations can be roughly divided into the transcription induction and the transcription repression. In an abstract model, the DNA elements for the transcription induction include an inducible promoter and a coding region of a protein.

An inducible promoter contains a DNA motif with a specific sequence (denoted as A) that can be recognized by its cognate transcription activator (TA) (denoted as TA(A)). When bound to a promoter, the TA triggers the transcription of the downstream gene. Using deterministic model from previous studies [6], such regulation can be modeled as follows:

$$
\frac{\mathrm{d}[M]}{\mathrm{d} t}=\alpha_{0}+\frac{\alpha_{1}[T A]^{n}}{K^{n}+[T A]^{n}}-k[M],
$$

where $[T A]$ and $[M]$ are the concentration of TA and messenger RNA (mRNA) of the regulated gene, respectively; $\alpha_{0}$ and $\alpha_{1}$ are the basal and maximum inducible promoter strengths, respectively; $K$ and $n$ are the apparent dissociation constant (specifying the affinity) and the Hill coefficient (specifying the cooperativity) of the TA-promoter binding, and $k$ is the degradation rate of mRNA.

When the rate of the translation and the mRNA degradation is not critical in design, the transcription and the translation can be combined into a simplified model:

$$
\frac{\mathrm{d}[P]}{\mathrm{d} t}=\alpha_{0}+\frac{\alpha_{1}[T A]^{n}}{K^{n}+[T A]^{n}}-k[P],
$$

where $[P]$ and $k$ are the concentration and the degradation rate of the protein product of the regulated gene, respectively.

The DNA elements for the transcription repression (include a constitutively active promoter, a transcription repressor (TR)-binding site and a coding sequence of the regulated gene. The TR-binding site is at the downstream of the promoter. It contains a DNA motif with a specific sequence and can be bound by the TR. When the TRbinding site is not bound by the TR, the promoter directs the mRNA synthesis. Similarly, this regulation can be modeled as

$$
\frac{\mathrm{d}[M]}{\mathrm{d} t}=\frac{\alpha_{1} K^{n}}{K^{n}+[T A]^{n}}-k[M],
$$

or

$$
\frac{\mathrm{d}[P]}{\mathrm{d} t}=\frac{\alpha_{1} K^{n}}{K^{n}+[T A]^{n}}-k[P] .
$$

We also use the post-translational regulation in the form of protease-mediated protein degradation and protein-protein interaction-based protein-inactivation. The biochemical model can be formulated as follows: (1) a protease triggers the degradation of its specific targets; (2) the specificity of a protease (target spectrum) is determined by designer; (3) a protease is inhibited when a protease inhibitor binds to it; (4) the specificity of a protease inhibitor is determined by designer.

A protease only affects the degradation rates of its target proteins. The model can be written as

$$
\frac{\mathrm{d}[P]}{\mathrm{d} t}=R_{\text {production }}-k_{1}[P]-\frac{k_{2}[P r]^{n}}{K^{n}+[P r]^{n}}[P]
$$

where $R_{\text {production }}$ is the production rate of the target protein, $[\mathrm{Pr}]$ is the concentration of the protease, $k_{1}$ is the rate constant of the basal degradation of the target protein, $k_{2}$ is the rate constant of the protease-mediated degradation of target protein, $K$ is the apparent dissociation constant and $n$ is the Hill coefficient of the proteaseprotein interaction.

The protease-protease inhibitor interaction can be expressed in the following scheme:

$$
\operatorname{Pr}^{*}+\operatorname{Pr} I^{*} \underset{k_{-1}}{\stackrel{k_{1}}{\leftrightarrows}} \operatorname{PrI} \cdot \operatorname{Pr} \stackrel{k_{2}}{\longrightarrow} \text { Degradation Product }
$$

where $\operatorname{Pr}^{*}$ is a free (unbound) protease and $\operatorname{PrI}^{*}$ is its free protease inhibitor. $P r I \cdot P r$ is the complex formed by the protease and its protease inhibitor. Assuming protein-protein interactions are much faster than transcriptions and translations. We can approximate this reaction at its steady state as follows:

$$
\frac{\left[\operatorname{Pr}^{*}\right]\left[\operatorname{PrI}^{*}\right]}{[\operatorname{PrI} \cdot \operatorname{Pr}]}=\frac{k_{-1}+k_{2}}{k_{1}}=K
$$

When the concentrations of both $\operatorname{Pr}$ and $\operatorname{PrI}$ are much greater than $K,\left[\mathrm{Pr}^{*}\right]$ can be approximated as

$$
\left[\operatorname{Pr}^{*}\right]=\left\{\begin{array}{l}
{[P r]-[P r I], \quad \text { if }[P r]>[P r I]} \\
0, \quad \text { otherwise }
\end{array},\right.
$$

where $[\mathrm{Pr}]$ is the concentration of the total $\mathrm{Pr}$ and $[\mathrm{PrI}]$ is the concentration of the total PrI. This reaction enables the substraction operation in biology systems.

\section{Detailed Design of FEBC}

In this section, we present the details of the three components of FEBC.

\section{A. Biological Clock}

We choose a repressilator [3] as the $\mathrm{BC}$ component in our design. The model of the repressilator can be written as a set of ordinary differential equations (ODEs) [3],

$$
\left\{\begin{array}{lll}
\frac{\partial M_{i}}{\partial T}=-\alpha_{M} M_{i}+\beta_{0}+\beta_{M} \frac{K_{P}^{2}}{K_{P}^{2}+P_{j}^{2}}, & i=1,2,3 \\
\frac{\partial P_{i}}{\partial T}=-\alpha_{P} P_{i}+\beta_{P} M_{i} & j=3,1,2
\end{array}\right.
$$

where

- $i$ and $j$ represent the identities of the gene and the associated protein product,

- $M_{i}$ is the mRNA concentration,

- $P_{i}$ is the protein concentration,

- $K_{P}$ is the apparent dissociation constant of the transcription factor-promoter interaction,

- $\alpha_{M}$ and $\alpha_{P}$ are the total degradation rates of the mRNA and the protein,

- $\beta_{0_{M}}$ and $\beta_{M}$ are the basal and maximum inducible promoter strengths.

The repressilator oscillation period is mainly determined by the degradation of the proteins $\alpha_{P}$ [3]. The Integrator adjusts the degradation rate in the FEBC.

\section{B. Bio Frequency Comparator (BFC)}

In this subsection, we discuss how to compare the frequencies of two signals. We then use network motifs [6], which are basic building blocks of biological systems, to design a BFC. 


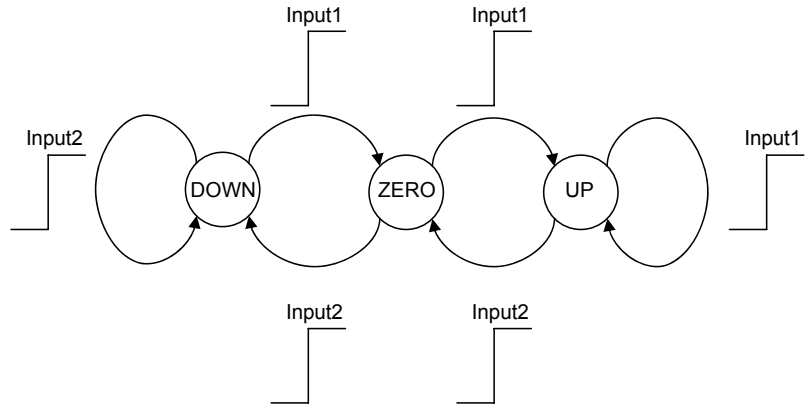

Figure 2. The state diagram of the BFC.

1) Frequency Comparing Mechanism: The BFC has two output signals UP and DOWN. The behavior of the BFC can be explained using the state diagram in Figure 2 The UP state means the UP signal is active and the DOWN signal is inactive. The DOWN state means the DOWN signal is active and the UP signal is inactive. The ZERO state means UP-DOWN is zero. Whenever the BFC sees a rising edge of INPUT1, it transits to a state to the right unless it is already in the rightmost state. Whenever the BFC sees a rising edge of INPUT2, it transits to a state to the left unless it is already in the leftmost state.

Using this mechanism, the BFC can tell the frequency difference between two signals. As shown in Figure 3, when the frequency of INPUT1 is greater than that of INPUT2, the BFC sees the rising edges of INPUT1 more frequently than those of INPUT2. Therefore, the state machine is mostly in the UP state. The UP signal is mostly active and the DOWN signal is inactive. Otherwise, the DOWN signal is mostly active and the UP signal is inactive. The more the frequency

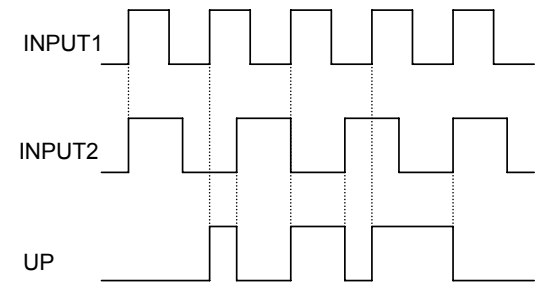

DOWN

Figure 3. Waveforms of the BFC, where the frequency of the INPUT1 is higher.

difference between the two signals, the more likelihood that the UP signal or the DOWN signal is active.

2) Designing BFC Using Network Motifs: Based on the previous discussion, the BFC shall have the following functions:

- It needs to detect the rising edges of the signals INPUT1 and INPUT2;

- It needs to remember a rising edge has come by keeping either the UP signal or the DOWN signal active;

- It needs to deactivate both the UP signal and the DOWN signal after the rising edges of both signals have come.

Biological systems are usually complex networks, which consist of many interacting chemical components, such as DNA, mRNA and proteins. In these networks, a number of patterns, called network motifs, have been found occurring much more frequently than those in random networks [7]. Since these motifs have specific functions and are conserved in evolution, they can be considered as basic building blocks of biological systems [6]. We use these motifs to design the BFC.

a) Pulse Generator: The rising edge of a signal can be recognized by the incoherent type-1 feed-forward loop (I1-FFL) motif [7] as shown in Figure 4 In this motif, the transcription factor $\mathrm{X}$ activates the transcriptions of the transcription repressor $\mathrm{Y}$ and the gene $\mathrm{Z}$, but the transcription repressor $\mathrm{Y}$ inhibits the transcription of $\mathrm{Z}$. When $\mathrm{X}$ is absent, $\mathrm{Y}$ is also absent. When the level of $\mathrm{X}$ increases, it takes a short period of time to increase $Y$ to the level enough to inhibit the production of $\mathrm{Z}$. Therefore, $\mathrm{Z}$ is briefly produced in this period of time. Then, the production of $\mathrm{Z}$ decreases. Thus, the level of $\mathrm{Z}$ first rises and then drops. Therefore, this motif is a pulse generator.

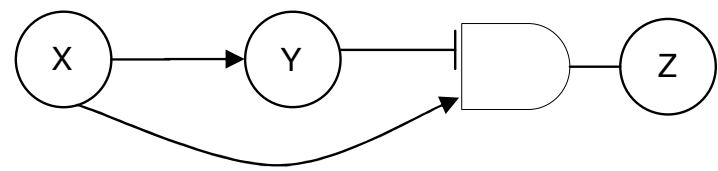

Figure 4. A pulse generator. The transcription factor $\mathrm{X}$ activates the transcriptions of the transcription repressor $\mathrm{Y}$ and the gene $\mathrm{Z}$, but the transcription repressor $\mathrm{Y}$ inhibits the transcription of $\mathrm{Z}$.

b) Memory and Delay Element: A 1-bit memory can be constructed by the positive autoregulation motif as shown in Figure 5 where the transcription factor $\mathrm{X}$ activates its own expression. When

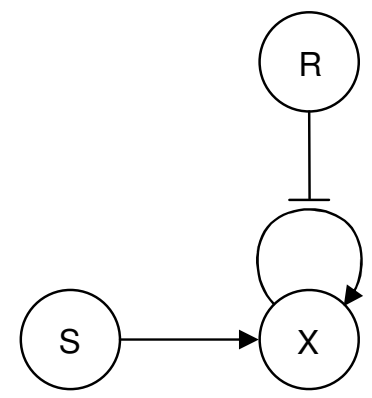

Figure 5. A strong positive autoregulation motif can be used a 1-bit memory. The transcription factor $\mathrm{X}$ activates its own expression. The transcription factors $\mathrm{S}$ sets the memory and the transcription factors $\mathrm{R}$ resets the memory.

the positive autoregulation is strong enough compared with the degradation rate of $\mathrm{X}$, the system is bi-stabile. Once the level of $\mathrm{X}$ is high, it is locked into the high expression state. The transcription factor $S$ also activates the production of $X$, which can set $X$ to the high expression state. The transcription factor $\mathrm{R}$ can inhibit the selfactivation of $\mathrm{X}$, which can set $\mathrm{X}$ to the low expression state.

When the self-activation is weak, the positive autoregulation motif can also serve as a delay element [6].

c) The Design of BFC: As shown Figure 6 we design the BFC by combining the above network motifs and some other components. The longer the delay through the transcription factor $\mathrm{Y}_{i}$, the stronger the pulse generated the pulse generator. Therefore, to make the pulse generator more robust, we add a delay element to each pulse generator. A strong positive feedback is added at the output of the pulse generator. The pulse will be remembered until it is reset though the resetter after the rising edges of both signals have come. We deliberately introduce some delay in the resetter by adding one more transcription factor R. Otherwise, the two memories may not be reset. The AND gate means that the resetter is activated only if both memories are set. 


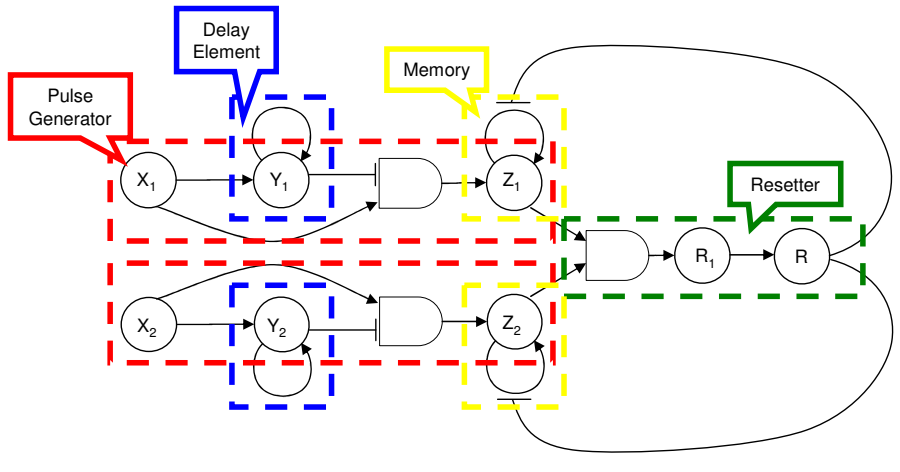

Figure 6. The design of the BFC. Each component is denoted by a dashed box and a callout box of the same color.

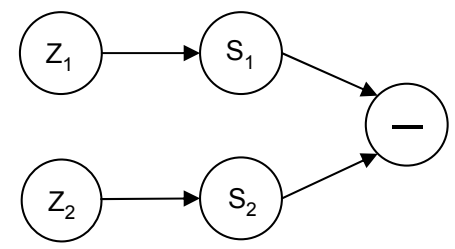

Figure 7. An integrator.

The model of the BFC can be written as

$$
\begin{aligned}
\frac{\mathrm{d} Y_{i}}{\mathrm{~d} t} & =-Y_{i}+\beta_{1}\left(X_{i}\right)+\frac{\beta_{2} Y_{i}^{2}}{\gamma_{1}^{2}+Y_{i}^{2}} \\
\frac{\mathrm{d} Z_{i}}{\mathrm{~d} t} & =-\alpha_{1}\left(Z_{i}-\frac{Z_{i}^{2}}{\gamma_{2}^{2}+Z_{i}^{2}} \frac{\beta_{3}}{1+R^{2}}-\frac{\beta_{4}\left(X_{i}\right)}{1+Y_{i}^{2}}\right) \\
\frac{\mathrm{d} R_{1}}{\mathrm{~d} t} & =-\alpha_{2}\left(R_{1}-\beta_{5} Z_{1} Z_{2}\right) \\
\frac{\mathrm{d} R}{\mathrm{~d} t} & =-\alpha_{3}\left(R-\frac{\beta_{6} R_{1}^{2}}{1+R_{1}^{2}}\right)
\end{aligned}
$$

where $\beta_{1}(X)$ and $\beta_{4}(X)$ are the functions describing the strength of the effects of the transcription factors $X_{i}$ on the transcriptions of $Y_{i}$. $\beta_{2}, \beta_{3}$ and $\beta_{6}$ are the maximum inducible promoter strength of the associated promoter. $\beta_{5}$ reflects the affinity between $Z_{1}$ and $Z_{2}$, the affinity between $Z_{1} \cdot Z_{2}$ complex and promoter, and the promoter strentgh. $\gamma_{1}$ and $\gamma_{2}$ are the corresponding apparent dissociation constants of transcription factor-promoter interactions. $\alpha_{1}, \alpha_{2}$ and $\alpha_{3}$ are some scaling factors.

\section{Integrator}

The last component of the FEBC is the Integrator, whose implementation is shown in Figure 7. The inputs $Z_{1}$ and $Z_{2}$ in Figure 7 are transcription factors that are the same as those in Figure 6 Since $\mathrm{Z}_{1}$ and $\mathrm{Z}_{2}$ have only two stable states, the model of the transcription of $S_{1}$ and $S_{2}$ can be written in a linear form

$$
\frac{\mathrm{d} S_{i}}{\mathrm{~d} t}=\eta Z_{i}
$$

where $\eta$ is a biochemical parameter. If $\mathrm{S}_{1}$ and $\mathrm{S}_{2}$ are a protease and its inhibitor, according to the discussions in Section III the free protease $S_{1}^{*}$ can be written as $S_{1}-S_{2}$, when $S_{1}>S_{2}$. Therefore, the production rate of the free form of the protease can be written as

$$
\frac{\mathrm{d} S_{1}^{*}}{\mathrm{~d} t}=\eta\left(Z_{1}-Z_{2}\right)
$$

or in the integration form

$$
S_{1}^{*}=\int_{-\infty}^{t} \eta\left(Z_{1}-Z_{2}\right) \mathrm{d} t .
$$

Therefore, this component preforms the integration of the difference of two signals biologically.

\section{Design VALidation and Biological IMPLEMENTATION OF FEBC}

\section{A. Validation of the FEBC Design}

By combining the components discussed in the previous section, we end up with the complete design of FEBC in Figure 8

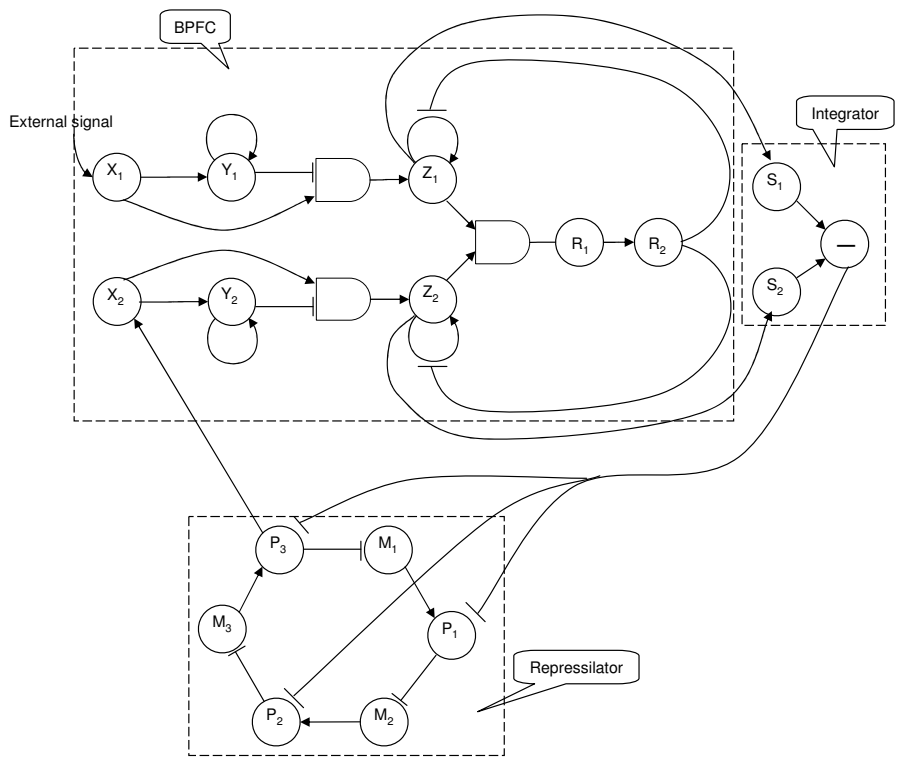

Figure 8. The complete design of FEBC is a combination of three components.

We have already described the model of the BFC (3) and the model of the Integrator (4). The output of the Integrator $S_{1}^{*}$ controls the frequency of the repressilator by

$$
\begin{aligned}
\frac{\partial M_{i}}{\partial t} & =-\alpha_{8}\left(M_{i}-\frac{\beta_{7}}{1+P_{j}^{2}}\right) & i & =1,2,3 \\
\frac{\partial P_{i}}{\partial t} & =-\alpha_{8}\left(\alpha_{7} P_{i}-M_{i}\right) & j & =3,1,2
\end{aligned},
$$

which is a different form of (2), where $\alpha_{7}$ is controlled by $S_{1}^{*}$ using the protease-protein interaction.

The transcription factor $\mathrm{P}_{3}$ activates the transcription of $\mathrm{X}_{2}$, which is an input of the BFC, by the model

$$
\frac{\mathrm{d} X_{2}}{\mathrm{~d} t}=-\left(X_{2}-\beta_{8} P_{3}^{4} /\left(\gamma_{3}^{4}+P_{3}^{4}\right)\right) .
$$

Using (3), (4), (5) and (6), we simulate the behavior or the BFC. In our simulation, we use a square wave with a duty ratio of $50 \%$ and an amplitude of 4 as the external input. We choose the parameter $\eta=0.00001$ in (4). Figure 9 shows the level of $S_{1}^{*}$ are eventually stabilized for three different input signal periods 70, 80 and 90 . Because $S_{1}^{*}$ controls the frequency of the internal signal, Figure 9 indicates that the frequency of the internal signal is also stabilized. We use Fast Fourier Transform (FFT) to analyze the frequency of the internal oscillation when $S_{1}^{*}$ is stabilized. The internal oscillation period is plotted against the period of the external signal in Figure 10 


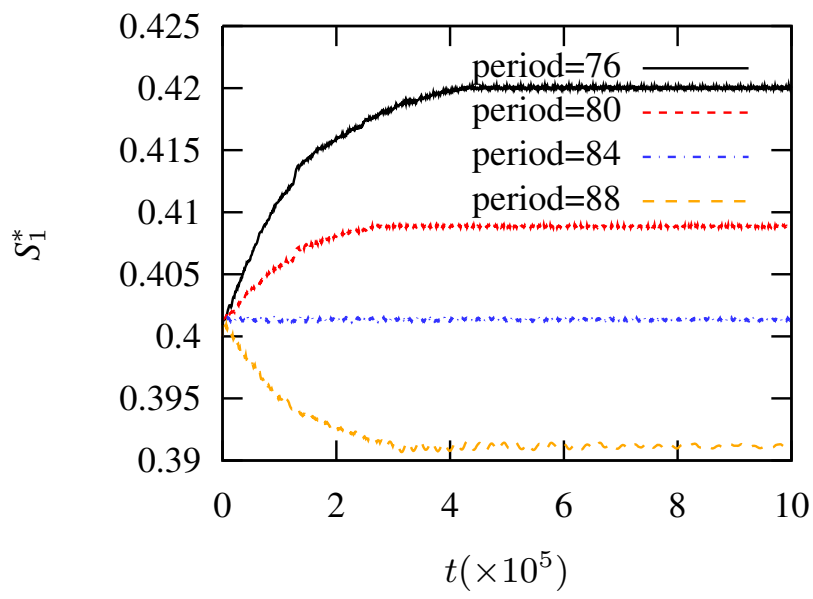

Figure 9. $\mathrm{S}_{1}^{*}$ is almost stabilized, so is the internal oscillatory frequency of the FEBC. $\alpha_{1}=1, \alpha_{2}=0.3, \alpha_{3}=0.3, \alpha_{7}=0.2 \frac{S_{1}^{* 2}}{1+S_{1}^{* 2}}, \alpha_{8}=2$, $\beta_{1}(X)=\frac{X^{2}}{1+X^{2}}, \beta_{2}=4, \beta_{3}=2.5, \beta_{4}(X)=0.5 \frac{X^{2}}{1+X^{2}}, \beta_{5}=0.25$,
$\beta_{6}=5, \beta_{7}=5, \beta_{8}=4, \gamma_{3}=3.16, \gamma_{1}=3$, and $\gamma_{2}=1$.

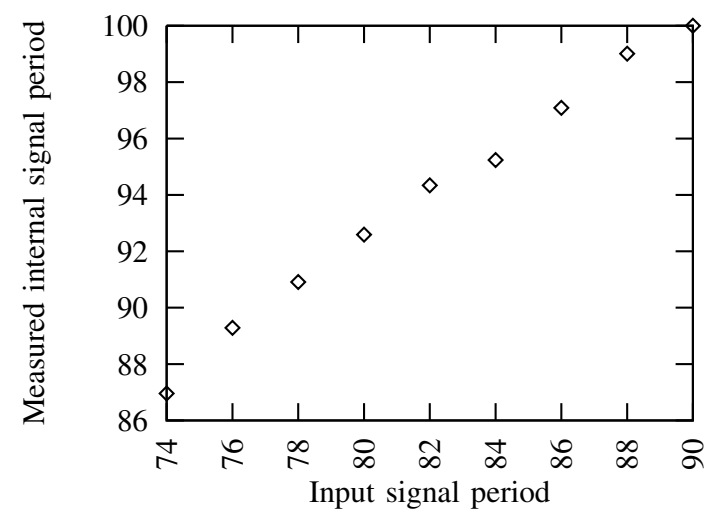

Figure 10. The period of the internal oscillation is controlled by the period of the external signal.

As we can see, the internal biological clock is entrained by the frequency of the external signal in our design of the FEBC.

We also use trapezoid waves as the input signals. We denote the rise time as $T_{1}$ and the period as $T$ (Figure 11 ). Figure 12 shows that

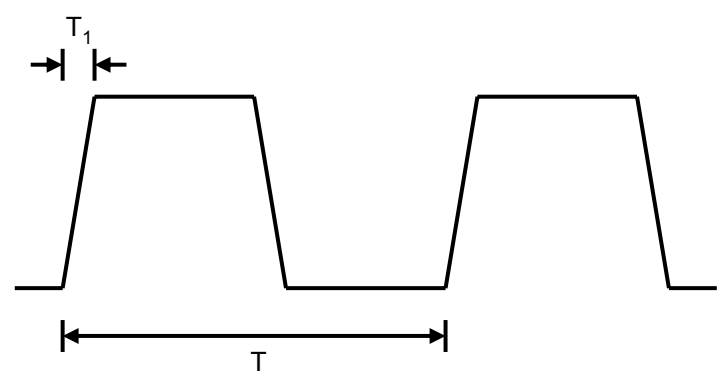

Figure 11. A trapezoid wave, whose period is $T$, takes time $T_{1}$ to rise from zero to the maximum.

the shape of input waveform also affect the frequency of the internal oscillation. When the slope of the rising edge reduces, the period of the biological clock also decreases. Therefore, both the period and the waveform of the external signal determine the period of the internal biological clock.

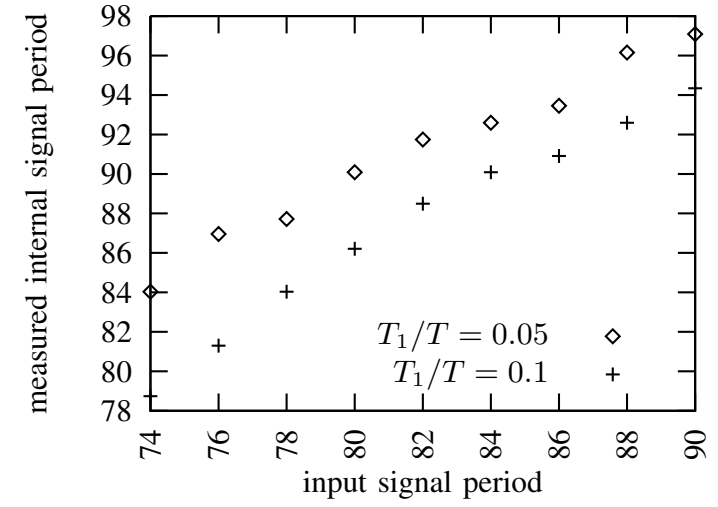

Figure 12. Not only the period of the external signal but also the actual waveform determine the period of the internal oscillation frequency. The sets of points are from two different trapezoid waveforms with $T_{1} / T=0.05$ and $T_{1} / T=0.1$.

\section{B. Biological Implementation}

A possible biological implementation is shown in Figure 13 Considering the emphasis of this paper, we do not specify the identity of proteins but use hypothetical proteins with the syntax ACTIVITY(SPECIFICITY) in the description of the genetic circuit. For example, the transcription activator which recognizes the inducible promoter $X$ is written as $T A(X)$; the protease which degrades the proteins $Y$ and $Z$ is written as $\operatorname{Pr}[Y, Z]$; and the inhibitor of this protease is written as $\operatorname{PrI}[\operatorname{Pr}[Y, Z]]$. It should be noted that our design is based on well established biochemical mechanisms, and is possible to be implemented in a biological system given the adequate molecular engineering efforts. Moreover, other mechanisms with similar kinetic properties can also be employed to implement the circuit (e.g. phosphorylation/dephosphorylation mechanism is an alternative of protease/inhibitor mechanism).

To begin with, the Repressilator Cassettes that consists of $T R(P 1)$, $T R(P 2), T R(P 3)$ and their corresponding repressor-binding sites act as the internal biological clock. The $T R(P 1)$-expressing cassette expresses $T A(X 2)$ in a bicistronic fashion, which serves as the internal input of the BPFC.

The external signal is a periodically changing concentration of the inducer (chemical compound) of $T A(X 1)$. The input signal is first converted to the transcription-activation activity of $T A(X 1)$ through allosteric regulation. The active form of $T A(X 1)$ triggers the transcription of its target genes in two multicistronic expression cassettes which are named as the Pulse Generator Cassettes. In the first cassette, the transcription of $T A(Y 1)$ and $T R(V 1)$ are driven by two inducible promoters $X 1$ and $Y 1$. $Y A(Y 1)$ may trigger its own expression through the weak positive autoregulation. In the second cassette, the transcription of $T A(Z 1)$ is induced by the active form of $T A(X 1)$, but is repressed by $T R(V 1)$ which is produced by the first cassette. With these Pulse Generator Cassettes, the expression of $T A(Z 1)$ is transiently stimulated upon the allostiric activation of $T A(X 1)$, but is soon repressed when $T R(V 1)$ accumulates to a certain level. $T A(Z 1)$, the output of the Pulse Generator Cassettes, activates the expressions of $T A(Z 1), T A(W 1)$ and $\operatorname{Pr}[P 1, P 2, P 3]$ in the third multicistronic cassette which is named as the Memory Cassette. Due to the bistability resulting from the strong positive autoregulation of $T A(Z 1)$, the transcription activity of this cassette will remain at a high level after it receives the pulse of $T A(Z 1) . T A(W 1)$ and $\operatorname{Pr}[T R(P 1), T R(P 2), T R(P 3)]$ produced by the Memory Cassette serves as the inputs of the Resetter Cassette and the Integrator, respectively. 


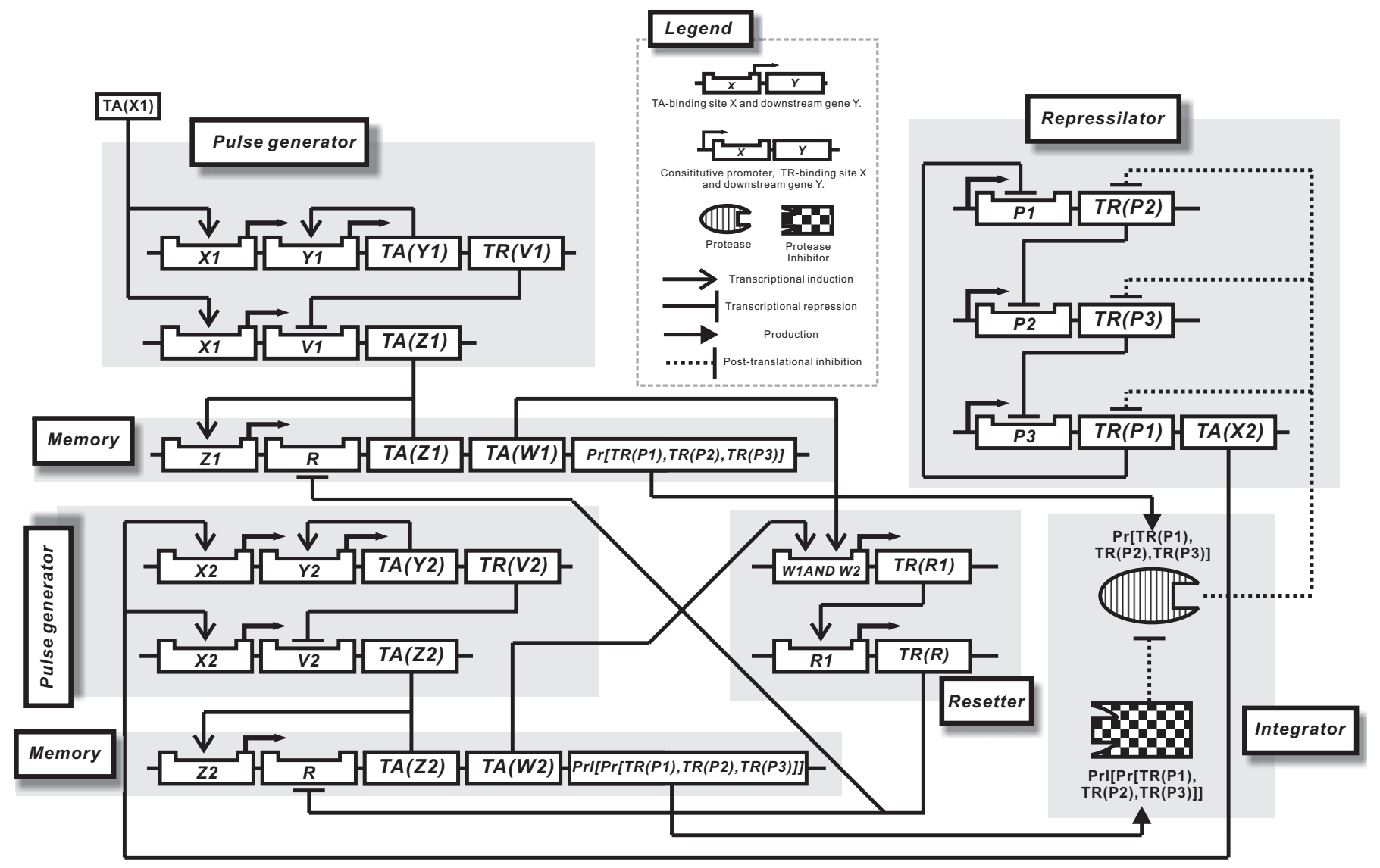

Figure 13. The biological implementation of FEBC.

Through another set of Pulse Generator Cassettes and its corresponding Memory Cassette (shown at the bottom left of Figure 13, the rising of the internal signal $T A(X 2)$ results in a stable, high-level transcription of $T A(W 2)$ and $\operatorname{PrI}[\operatorname{Pr}[T R(P 1), T R(P 2), T R(P 3)]]$.

$T A(W 1)$ and $T A(W 2)$ are two subunits of a TA which is only active when $T A(W 1)$ and $T A(W 2)$ form a heterodimer. In the Resetter Cassette, this dimeric TA induces the transcription of $T A(R 1)$ which in turn induces the expression of $T R(R) . T R(R)$ represses the transcription of both Memory Cassettes and sets their transcriptional activity to a low level so that they are ready to accept the next risings of the external and the internal inputs.

The Integrator consists of the protease $\operatorname{Pr}[T R(P 1), T R(P 2), T R(P 3)]$ and its protease inhibitor $\operatorname{Pr}[\operatorname{Pr}[T R(P 1), T R(P 2), T R(P 3)]]$. The protease $\operatorname{Pr}[T R(P 1), T R(P 2), T R(P 3)]$ accelerates the degradation of the TRs in Repressilator, which shortens the period of Repressilator; in contrast, its inhibitor $\operatorname{Pr}[\operatorname{Pr}[T R(P 1), T R(P 2), T R(P 3)]]$ increases the period.

The consequence of all the above mechanisms ensures the frequency of the internal oscillator to be controlled by the frequency of the external signal.

\section{CONCLUSiONS}

In this work, we design a genetic circuit that can adjust the frequency of its internal oscillator according to the frequency of the external signal. The functionality is confirmed by the analysis of the circuit using ordinary differential equations (ODEs) based on the deterministic model. As the main purpose of this work is to set an example of how to adapt a concept in electronic engineering to a biological system, we simplify the simulation model and do not take noise of various origins (e.g. variation of nutrient, $\mathrm{pH}$ and temperature of culture media; cell division; stochasticity of transcription and translation) into account. Future studies would involve designing the biological clock entrained by both the phase and the frequency of the input signal, making the designs more robust with respect to parameter variations and noise, and finally implementing the design in a biological system.

\section{REFERENCES}

[1] D. Bell-Pedersen, V. M. Cassone, D. J. Earnest, S. S. Golden, P. E. Hardin, T. L. Thomas, and M. J. Zoran, "Circadian rhythms from multiple oscillators: Lessons from diverse organisms," Nat Rev Genet, vol. 6, no. 7, pp. 544-556, Jul. 2005.

[2] M. W. Young, "The molecular control of circadian behavioral rhythms and their entrainment in drosophila," Annual Review of Biochemistry, vol. 67, pp. 135-152, Jul. 1998.

[3] M. B. Elowitz and S. Leibler, "A synthetic oscillatory network of transcriptional regulators," Nature, vol. 403, pp. 335-338, Jan. 2000.

[4] M. R. Atkinson, M. A. Savageau, J. T. Myers, and A. J. Ninfa, "Development of genetic circuitry exhibiting toggle switch or oscillatory behavior in escherichia coli." Cell, vol. 113, no. 5, pp. 597-607, May 2003.

[5] F. M. Gardner, Phaselock Techniques, 3rd ed. Wiley-Interscience, Jul. 2005.

[6] U. Alon, An Introduction to Systems Biology: Design Principles of Biological Circuit, 1st ed. Chapman \& Hall/CRC, 2006.

[7] R. Milo, S. Shen-Orr, S. Itzkovitz, N. Kashtan, D. Chklovskii, and U. Alon, "Network motifs: simple building blocks of complex networks," Science, vol. 298, no. 5594, pp. 824-827, Oct. 2002. 